\title{
The Dispersionless Lax Equations and Topological Minimal Models
}

\author{
I. Krichever \\ Landau Institute for Theoretical Physics, USSR Academy of Sciences, \\ Kosygina 2, SU-117940 Moscow, USSR
}

Received April 15, 1991

\begin{abstract}
It is shown that perturbed rings of the primary chiral fields of the topological minimal models coincide with some particular solutions of the dispersionless Lax equations. The exact formulae for the tree level partition functions of $A_{n}$ topological minimal models are found. The Virasoro constraints for the analogue of the $\tau$-function of the dispersionless Lax equation corresponding to these models are proved.
\end{abstract}

\section{Introduction}

Topological minimal models were introduced in [1], following general considerations in [2]. They are a twisted version of the discrete series of $N=2$ superconformal Landau-Ginzburg (LG) models. A large class of $N=2$ superconformal LG models have been studied in [3]. It was shown that a finite number of states are topological which means that their operator products have no singularities. These states form a closed ring. For the LG models these rings of the primary chiral fields take the particular simple form and can be represented in terms of the corresponding superpotentials. In the topological twisted version of the superconformal LG models the chiral primary fields are the only physical excitations.

In [4] some evidence was provided that the topological minimal models coupled with topological $2-d$ gravity are equivalent to multi-matrix models. (After $[2,6]$ it was recognized in [5] that the multi-matrix model corresponds to topological gravity coupled to some matter system, although precisely which system was unknown.) On the other hand the multi-matrix model is believed to be equivalent to ordinary matter coupled to $2-d$ gravity. The last equivalence has led to remarkable connections between the non-perturbative theory of $2-d$ gravity and the theory of the integrable soliton equations [7-9].

Our work was stimulated by the results of [10], where the perturbed rings of the primary chiral fields were found. All correlation functions can be easily obtained 
with the help of the perturbed superpotentials. Moreover, the deep connections of the perturbed rings with some algebraic truncation of the Lax equations were found. In this paper we shall show that the perturbed chiral primary rings of the topological minimal models correspond to particular solutions of the so-called dispersionless Lax equations which are the quasi-classical limit of the ordinary Lax equations.

Roughly speaking the quasi-classical limit corresponds to the solutions which slowly depend on the variables $x, t$. For the $\mathrm{KdV}$ equation the quasi-classical limit has the form

$$
u_{t}=\frac{3}{2} u u_{x}
$$

(i.e. the form of the $\mathrm{KdV}$ equation but without dispersion term). As it was noticed in [11], the dispersionless Lax equations and their two-dimensional generalizations (the dispersionless KP equation is called Khokhlov-Zabolotskaya equation) are the particular cases (the genus $g=0$ case) of the general Whitham equations for the algebraic-geometrical solutions of a two-dimensional integrable system. Therefore, the general construction of the exact solutions of the Whitham equations which was proposed in [11] can be applied to the dispersionless Lax equation.

The organization of this paper is as follows. In Sect. 2 we define the hierarchy of the dispersionless Lax equation in the form which follows from [11] and give in closed form the construction of their exact solutions. In Sect. 3 we review the results of [10] and prove that the perturbed superpotential of the minimal $A_{n}$ models coincide with some particular solutions of the first $n$ flows of the dispersionless Lax equation. At the end of this section the exact formula for the partition of the perturbed topological minimal $A_{n}$ model will be obtained. It turns out that it coincides with some special $\tau_{k}$-function of the dispersionless Lax equation if we consider only $n$ first flows of the corresponding hierarchy. The Virasoro constraints for this $\tau_{k}$-function depending on all times will be proved in Sect. 4. In the last section we review some of the main ideas of the algebraicgeometrical perturbation theory of the two-dimensional integrable systems and consider within the framework of this theory the Khokhlov-Zabolotskaya hierarchy. (We would like to mention here two papers which are devoted to the dispersionless Lax and KP equations. In [15] the construction of integrals for dispersionless Lax equations was proposed. In [16] the construction of particular solutions for Khokhlov-Zabolotskaya equation was presented.)

\section{The Dispersionless Lax Equations}

For each integer $n \geqq 2$ the $n^{\text {th }}$ reduction of the KP hierarchy can be written as a system of the compatible evolution equations on the coefficients of the ordinary differential operator

$$
L=\partial^{n}+u_{n-2} \partial^{n-2}+\ldots+u_{0}, \quad \partial=\frac{\partial}{\partial x} .
$$

These equations are equivalent to the operator equations

$$
\partial_{i} L=\left[\left[L^{\frac{i}{n}}\right]_{+}, L\right], \quad \partial_{i}=\frac{\partial}{\partial t_{i}}, \quad i=1,2, \ldots,
$$

where []$_{+}$denotes the differential part of a pseudo-differential operator. 
Now we are going to define the quasi-classical limit of (2.2) as a system of evolution equations on the coefficients of the polynomial $E(p)$

$$
E(p)=p^{n}+u_{n-2} p^{n-2}+\ldots+u_{0} .
$$

At the beginning we shall present them in the form (2.5) which was obtained in [11] and which can be used for the construction of their solutions. Later we shall show that they are equivalent to the evolution equations (2.10) which are the direct quasi-classical limit of Lax equations (2.2).

Let's define the set of the polynomials $\Omega_{i}(p)$ by the following formula:

$$
\Omega_{j}(p)=\left[E^{\frac{i}{n}}(p)\right]_{+},
$$

which means that $\Omega_{i}(p)$ is equal to the non-negative part of the Laurent expansion of the fractional power of $E(p)$. The coefficients of $\Omega_{i}(p)$ are the polynomial functions of the coefficients of the polynomial $E(p)$.

Let's assume that the coefficients $u_{i}$ are the functions of the variable $x, u_{i}=u_{i}(x)$. Then the dispersionless Lax hierarchy is by definition the set of the evolution equation on $u_{i}(x)$, which are equivalent to the following equations

$$
\partial_{i} p(E)=\partial_{x} \Omega_{i}(E) \text {. }
$$

[Here and sometimes below we omit the notion of a dependency of $p, \Omega$ on the variables $t_{i}$, i.e. $p(E)=p\left(E, t_{i}\right)$ and so on.]

From (2.4) it follows that $p=\Omega_{1}(p)$. Hence, the variable $x$ and $t_{1}$ can be identified.

The meaning of Eqs. (2.5) is as follows. By definition $E$ is a polynomial in the váriable $p$. The inverse function $p(E)$ can be expanded at the infinity

$$
p(E)=K+\xi_{1} K^{-1}+\ldots, \quad K^{n}=E .
$$

The polynomials $\Omega_{j}$ can be also considered as the functions of the variable $E$ after substitution $\Omega_{i}(E)=\Omega_{i}(p(E))$. They have the expansion

$$
\Omega_{i}=K^{i}+\chi_{i, 1} K^{-1}+\ldots
$$

In (2.5) the derivatives with respect to $x, t_{i}$ are considered for the fixed $E$ (or $K$ ).

The coefficients of the expansion (2.7) are the polynomials in $u_{i}$. Therefore, Eqs. (2.5) are equivalent to an infinite system of the equations for the functions $u_{i}$. We are going to show that only a finite number of them are independent, i.e. that Eqs. (2.5) define the evolution equation for the polynomial $E(p)$.

The partial derivatives with respect to $x, t_{i}$ for the fixed $E$ or $p$ are related to each other in the following way:

$$
\begin{gathered}
\partial_{x} \Omega_{i}(E)=\partial_{x} \Omega_{i}(p)-\frac{d \Omega_{i}}{d E} \partial_{x} E(p), \\
\partial_{i} p(E)=-\frac{d p}{d E} \partial_{i} E(p) .
\end{gathered}
$$

Hence, from $(2.5,2.8,2.9)$ it follows that

$$
\partial_{i} E(p)=\frac{d \Omega_{i}}{d p} \partial_{x} E(p)-\frac{d E}{d p} \partial_{x} \Omega_{i}
$$


In order to prove that $(2.10)$ is well-defined evolutional equation for $E(p)$ it is necessary to show that the right-hand side of (2.10) is a polynomial in $p$ of degree less than $n-1$. From the definition of $\Omega_{i}$ it follows that

$$
\Omega_{i}(p)=E^{\frac{i}{n}}(p)-\left[E^{\frac{i}{n}}(p)\right]_{-},
$$

where

$$
\left[E^{\frac{i}{n}}(p)\right]_{-}=O\left(p^{-1}\right)
$$

is the negative part of the fractional power of the polynomial $E(p)$. Substitution of (2.11) into (2.10) gives that

$$
\partial_{i} E=\frac{d E}{d p} \partial_{x}\left[E^{\frac{i}{n}}\right]--\frac{d\left[E^{\frac{i}{n}}\right]-}{d p} \partial_{x} E
$$

which proves that (2.10) is well-defined equation.

The Eqs. (2.5) have the form of the compatibility conditions for the equations

$$
\Omega_{i}(E)=\partial_{i} S(E), \quad i=1,2, \ldots .
$$

This observation is trivial but very useful for the construction of the exact solutions.

For each fixed positive integer $m$ let's define the polynomial

$$
S_{m}^{+}\left(p, t_{1}, \ldots, t_{m}\right)=\sum_{i=1}^{m} t_{i} \Omega_{i}(p, t)
$$

These polynomials are the partial sums of the formal series

$$
\begin{gathered}
S_{\infty}^{+}\left(p, t_{1}, \ldots\right)=\sum_{i=1}^{\infty} t_{i} \Omega_{i}(p), \\
S_{m}^{+}\left(p, t_{1}, \ldots, t_{m}\right)=S_{\infty}^{+}\left(p, t_{1}, \ldots, t_{m}, 0,0, \ldots\right) .
\end{gathered}
$$

The coefficients of this formal series are well-defined as the formal series in the variables $t_{i}$. We shall return to it later in Sect. 4 .

Up to now the variables $t_{i}$ and $u_{1}, \ldots, u_{n-2}$ can be considered as the independent variables. Let's define the dependence of the polynomial $E(p)$ with respect to the variables $t_{1}, \ldots, t_{m}, m>n$, with the help of the following relations:

$$
\frac{d S_{m}^{+}}{d p}(p)=B_{m}(p) \frac{d E}{d p}(p),
$$

where $B_{m}(p)$ should be some polynomial in respect to $p$

$$
B_{m}=b_{m-n} p^{m-n}+\ldots+b_{0} .
$$

The polynomial equation (2.17) is equivalent to the system of $m$ equations for the unknown functions $u_{i}$ and $b_{j}$. The first $n-m+1$ of them define the coefficients $b_{j}$ of the polynomial $B_{m}(p)$ as the rational functions of $u_{i}$ and $t_{i}$. The last $n-1$ equations define $u_{i}$ as the algebraic functions of the variables $t_{1}, \ldots, t_{m}$, i.e.

$$
u_{i}=u_{i}\left(t_{1}, \ldots, t_{m}\right) \text {. }
$$


Remark. From the defining relations (2.17) it immediately follows that $u_{i}$ don't depend on $t_{n}, t_{2 n}, \ldots$.

Theorem [11]. If the functions $u_{i}\left(t_{1}, \ldots, t_{m}\right)$ are defined with the help of the relations (2.17) then they are solutions of the dispersionless Lax equations (2.5) (or equivalently (2.10)).

Remark. This is a very particular case of the construction [11]. But we restrict our consideration to this case because of its connection with the topological minimal models.

Let's give the proof of this theorem. From (2.15) it follows that the expansion of the polynomial $S_{m}^{+}(E)$ has the form

Therefore,

$$
S_{m}^{+}=\sum_{i=1}^{m} t_{i} K^{i}+O\left(K^{-1}\right), \quad K^{n}=E .
$$

$$
\partial_{i} S_{m}^{+}(E)=K^{i}+O\left(K^{-1}\right)=\Omega_{i}(E)+O\left(K^{-1}\right) .
$$

The next step is the proof that from (2.17) it follows that $S_{m, i}^{+}(E)$ which is equal to the left-hand side of (2.19) is a polynomial in $p$. Let's consider this derivative $S_{m, i}^{+}(E)$ as function on the whole complex $p$-plane. It's holomorphic everywhere except at the points $q_{s}$ which are the zeros of the differential

$$
d E\left(q_{\mathrm{s}}\right)=0, \quad s=1, \ldots, n-1 .
$$

(For simplicity we consider the generic case where all zeros of $d E$ are simple.) In the neighbourhood of this point the local coordinates are

$$
\sqrt{E-E_{s}}, \quad E_{s}=E\left(q_{s}\right) .
$$

Hence, a priori $S_{m}^{+}(E)$ has the expansion

$$
S_{m}^{+}(E)=\alpha_{s} \sqrt{E-E_{s}}+\beta_{s}\left(E-E_{s}\right)+\ldots,
$$

and consequently the derivative $S_{m, i}^{+}(E)$ might be singular at the points $q_{s}$. But the defining relations (2.17) are equivalent to the equalities

$$
d S_{m}^{+}\left(q_{s}\right)=0, \quad s=1, \ldots, n-1,
$$

which imply $\alpha_{s}=0$. Therefore, the derivative $S_{m, i}^{+}(E)$ is regular everywhere except at the infinity. Then from (2.19) it follows that

$$
\partial_{i} S_{m}^{+}(E)=\Omega_{i}(E)=\Omega_{i}(p) .
$$

For $i=1$ we have

$$
\partial_{x} S_{m}^{+}(E)=p(E) .
$$

The compatibility conditions of (2.22) and (2.23) are exactly the dispersionless Lax equations.

\section{The Perturbed Primary Chiral Rings of the Topological Minimal Models}

At the beginning of this section we recall the results of [10], where the perturbed rings of primary chiral fields were found. The construction of these rings which was proposed in [10] is very similar to the construction of the solutions for the 
dispersionless Lax equation which was given in the previous section. That's why it is not so surprising that these two constructions give the same answer.

Topological field theories were introduced by Witten $[2,12]$. These theories possess a nilpotent symmetry $Q, Q^{2}=0$, and the physical observables are the cohomology classes of the operator $Q$. The stress-energy tensor $T$ is $Q$-exact. Therefore, the correlation functions of the observables are independent on the twodimensional metric. The topological conformal field theories (TCFT) are the special class of topological field theories in which $T$ is traceless even before restricting to the $Q$-cohomology. For each physical operator $\Phi_{i}$ in TCFT there are three types of observables

$$
\Phi_{i, 0}(P), \quad \int_{C} \Phi_{i, 1}, \quad \int_{\Sigma} \Phi_{i, 2},
$$

where $\Phi_{i, 0}, \Phi_{i, 1}, \Phi_{i, 2}$ are zero-, one-, two-forms, respectively; $P$ is a point and $C$ is a contour on the surface $\Sigma$. The different components $\Phi_{i, 0}, \Phi_{i, 1}, \Phi_{i, 2}$ of superfield $\Phi_{i}$ are related by the descendent equations [2]

$$
\begin{aligned}
& d \Phi_{i, 0}=\left\{Q, \Phi_{i, 1}\right\}, \\
& d \Phi_{i, 1}=\left\{Q, \Phi_{i, 2}\right\} .
\end{aligned}
$$

Below we shall consider only the correlation function of zero- and two-form operators. For simplicity, the notations of weights of forms will be omitted. In the following all integrated operators will be understood to be two-forms. The correlation functions of zero-form operators

$$
\left\langle\prod_{s=1}^{k} \Phi_{k}\left(P_{k}\right)\right\rangle
$$

do not depend on the points $\left(P_{S}\right)$. They can be represented in terms of the threepoint functions

$$
c_{i, j, k}=\left\langle\Phi_{i} \Phi_{j} \Phi_{k}\right\rangle
$$

For example,

$$
\left\langle\Phi_{i} \Phi_{j} \Phi_{k} \Phi_{l}\right\rangle=\sum_{m} c_{i j}^{m} c_{m k l}
$$

where the matrix

$$
\eta_{i j}=\left\langle\Phi_{i} \Phi_{j}\right\rangle
$$

is used to raise the index. The self-consistency of the factorisation (3.5) implies that the operator algebra of the fields $\Phi_{i}$ given by

$$
\Phi_{i} \Phi_{j}=\sum_{k} c_{i j}^{k} \Phi_{k}
$$

is associative. In $N=2$ theories this algebra is known as the chiral primary $\operatorname{ring} R$. As it was shown in [3] the ring $R$ can be exactly described in terms of the superpotential $W\left(p_{i}\right)$ of the corresponding LG model. The LG description of the $A_{n}$ minimal models uses only a single LG field $p$ and in that of the other models there are two fields $p_{i}$. The ring $R$ is isomorphic to the ring of polynomials in the LG fields $p_{i}$, modulo the equation of motion

$$
\frac{\partial}{\partial p_{i}} W\left(p_{i}\right)=0, \quad R=\frac{C\left[p_{i}\right]}{d W\left(p_{i}\right)=0} .
$$


The potential for different series is given by

$$
\begin{aligned}
A_{n}: W(p) & =p^{n+1}, \\
D_{n}: W(p, q) & =p^{n+1}+p q^{2}, \\
E_{6}: W(p, q) & =p^{3}+q^{4}, \\
E_{7}: W(p, q) & =p^{3}+p q^{3}, \\
E_{8}: W(p, q) & =p^{3}+q^{3} .
\end{aligned}
$$

For the $A_{n}$ series which will be considered below the chiral primary fields are identified with $\Phi_{i}=p^{i}, i=0,1, \ldots, n-1$, and generate the ring

$$
\begin{array}{ll}
\Phi_{i} \Phi_{j}=\Phi_{i+j}, & i+j<n, \\
\Phi_{i} \Phi_{j}=0, & i+j>n-1 .
\end{array}
$$

Hence, all correlation functions of the zero-form operators can be found exactly. In [10] it was shown for any TCFT that the correlation functions of the zero-form operators and of the integrals of the two-form operators can be factorized and expressed in terms of the correlation functions which contain only three zero-form operators, i.e. through correlation functions of the form

$$
c_{i j k, l m \ldots s}=\left\langle\Phi_{i} \Phi_{j} \Phi_{k} \int \Phi_{l} \int \Phi_{m} \ldots \int \Phi_{s}\right\rangle .
$$

Moreover, it was shown that the insertion of the integral of two-form operator acts as the derivative and, therefore, (3.11) can be represented in form

$$
c_{i j k, l m \ldots s}=\left.\partial_{l m \ldots s} c_{i j k}\left(t_{0}, t_{1}, \ldots, t_{n-2}\right)\right|_{t=0},
$$

where $c_{i j k}(t)$ are the three point correlation functions of the perturbed primary chiral ring. The parameters $t_{i}$ are the coupling constants of the physical fields. They provide a parametrization of the space of topological models which are described as the deformation of the initial TCFT.

In the case of the topological minimal models the deformed chiral ring can be described in terms of the deformed superpotential $W\left(p, t_{0}, \ldots, t_{n-2}\right)$. The threepoint correlation functions of the perturbed ring are given by the relation

$$
\Phi_{i}(p, t) \Phi_{j}(p, t)=\sum_{k} c_{i j}^{k}(t) \Phi_{\kappa}(p, t)\left(\bmod \partial_{p} W(p, t)\right) .
$$

Now we are going to describe, according to [10], the equations which define uniquely the dependence of $W$ on the coupling constants.

For any polynomial $W$ in the ring

$$
R=C[p] /(d W(p)=0)
$$

there exist the natural inner product

$$
\left\langle\Phi_{i} \Phi_{j}\right\rangle=\operatorname{res}_{\infty}\left(\frac{\Phi_{i} \Phi_{j}}{\partial_{p} W} d p\right) .
$$

For given $W$ the primary fields $\Phi_{i}$ can be uniquely defined with the help of the orthogonality conditions $\Phi_{i}$

$$
\left\langle\Phi_{i} \Phi_{j}\right\rangle=\eta_{i j}=\delta_{i+j, n-2},
$$


where $n$ is the degree of $W$ (we consider the deformations of the $A_{n-1}$ minimal topological model) and with the help of normalizing conditions

$$
\Phi_{i}(p, t)=p^{i}+O\left(p^{i-1}\right) .
$$

Then the $W(p, t)$ can be found (in principle) from the equations

$$
\Phi_{i}(p, t)=-\partial_{i} W(p, t)
$$

and from the initial conditions

$$
W(p, 0)=\frac{p^{n}}{n}
$$

(we use the same change of the normalization of $W$ as in [10] for easier comparison of the results which were obtained in [10] and in previous section of this work).

The orthogonality conditions $(3.15,3.16)$ were solved in $[10]$ in the exact form

$$
\Phi_{i}=\frac{1}{i+1} \frac{d}{d p}\left[(n W)^{\frac{i+1}{n}}\right]_{+},
$$

where []$_{+}$denotes, as before, the non-negative part of the Laurent series. After substitution of (3.19) into (3.17) one shall obtain the equations for $W$ in closed form. They were exactly solved in [10].

Instead of presenting this solution here we are going to show its coincidence with the solutions of the dispersionless Lax equations which were obtained in the previous section.

Let $E\left(p, t_{1}, \ldots, t_{n-1}, t_{n+1}\right)$ be the solution of the dispersionless Lax equations which was constructed in the previous section (it means that in the construction we have to consider the case $m=n+1$ ). Then the superpotential of the perturbed $A_{n-1}$ topological model is equal to

$$
n W\left(p, t_{0}, \ldots, t_{n-2}\right)=E\left(p, t_{0}, \frac{t_{1}}{2}, \ldots, \frac{t_{n-2}}{n-1}, \frac{1}{n+1}\right) .
$$

From (3.20) and (3.19) it follows that after the change of variables

$$
t_{i} \rightarrow \frac{t_{i-1}}{i}
$$

the primary chiral fields coincide with

$$
\Phi_{i}=\frac{1}{i+1} \frac{d}{d p} \Omega_{i+1},
$$

where $\Omega_{i}$ are given by the formula (2.4). Therefore, for the proof of (3.20) it is enough to show that

$$
\frac{d}{d p} \Omega_{i}\left(p, t_{1}, \ldots, t_{n+1}\right)=-\frac{1}{n} \partial_{i} E\left(p, t_{1}, \ldots, t_{n+1}\right) .
$$

From (3.22) we have

$$
\Omega_{i}(p)=\Omega_{i}(E)=\partial_{i} S_{n+1}^{+}(E)=\partial_{i} S_{n+1}^{+}(p)-\frac{d S_{n+1}^{+}}{d E} \partial_{i} E(p) .
$$

For $m=n+1$ the defining relations (2.17) have the form

$$
\frac{d S_{n+1}^{+}}{d E}=\frac{1}{n} p
$$


Hence,

$$
n S_{n+1}^{+}=\int p d E=p E-\int E d p .
$$

After substitution of $(3.24,3.25)$ into (3.22) we shall obtain (3.22). Therefore the equality (3.20) is proved.

As it was shown in [10] all information about the correlation functions of the zero- and two-form operators is encoded in the partition function of the perturbed topological model (at the tree-level), because

$$
c_{i j k}(t)=\partial_{i} \partial_{j} \partial_{k} F(t) \text {. }
$$

The equalities (3.26) can be considered as the defining relations for $F$. In [10] they were integrated twice and the following formula for the derivatives of $F$ was obtained:

$$
\partial_{i} F=\operatorname{res}_{\infty}\left(\frac{(n W)^{\frac{n+1+i}{n}}}{(i+1)(n+i+1)} d p\right) .
$$

The last integration was not performed. We are going to prove the following exact formula for the tree-level partition function

$$
F=-\frac{1}{2} \operatorname{res}_{\infty}\left(S_{n+1}^{-} d S_{n+1}^{+}\right),
$$

where $S_{n+1}^{+}$is the non-negative and $S_{n+1}^{-}$is the negative parts of the Laurant series

$$
S_{n+1}=\frac{1}{n+1} K^{n+1}+\sum_{i=0}^{n-2} \frac{t_{i}}{i+1} K^{i+1}
$$

where

$$
K^{n}(p, t)=n W(p, t)
$$

(After the change of variables

$$
t_{i} \rightarrow \frac{t_{i-1}}{i}
$$

$S_{n+1}^{+}$will coincide with the polynomial which was used in previous section.)

From (3.24) it follows that (3.27) can be transformed into the following form:

$$
\begin{aligned}
\partial_{i} F & =\operatorname{res}_{\infty}\left(\frac{K^{n+1+i}}{(i+1)(n+1+i)} n d \frac{d S_{n+1}^{+}}{d K^{n}}\right) \\
& =-\operatorname{res}_{\infty}\left(\frac{K^{i+1}}{i+1} d S_{n+1}^{+}\right)=-\operatorname{res}_{\infty}\left(\frac{\left[K^{i+1}\right]_{-}}{i+1} d S_{n+1}^{+}\right) .
\end{aligned}
$$

Let's find the derivatives $F_{1, i}$, where $F_{1}$ is equal to the right-hand side of (3.28). The residue of the differential does not depend on the choice of the local parameter. We can use $K(p)$ as the local parameter. Then, as it was shown in the previous section

$$
\partial_{i} S_{n+1}^{+}(K)=\frac{\left[K^{i+1}\right]_{+}}{i+1}=\frac{\Omega_{i+1}}{i+1} .
$$

From the definition of $S_{n+1}$ it follows

$$
\partial_{i} S_{n+1}(K)=\frac{1}{i+1} K^{i+1} .
$$


Hence,

$$
\partial_{i} S_{n+1}^{-}(K)=\frac{1}{i+1}\left[K^{i+1}\right]_{-} .
$$

From (3.32) and (3.33) we have

$$
\partial_{i} F_{1}=-\frac{1}{2(i+1)} \operatorname{res}\left(\left[K^{i+1}\right]_{-} d S_{n+1}^{+}-\left[K^{i+1}\right]_{+} d S_{n+1}^{-}\right) \text {. }
$$

The last equality and the identity

$$
\begin{aligned}
0 & =\operatorname{res}\left(K^{i+1} d S_{n+1}\right) \\
& =\operatorname{res}\left(\left[K^{i+1}\right]_{+} d S_{n+1}^{-}+\left[K^{i+1}\right]_{-} d S_{n+1}^{+}\right)
\end{aligned}
$$

prove that

$$
\partial_{i} F_{1}=-\operatorname{res}_{\infty}\left(\frac{\left[K^{i+1}\right]_{-}}{i+1} d S_{n+1}^{+}\right) .
$$

Hence, $F=F_{1}$ and the formula (3.28) is proved.

\section{The Virasoro Constraints}

In this chapter we restore the "times" $t_{i}$ as they were in Sect. 2. Let's consider the function $F\left(t_{1}, t_{2}, \ldots\right)$

$$
F=-\frac{1}{2} \operatorname{res}\left(S^{-} d S^{+}\right)=-\frac{1}{2} \operatorname{res}\left(S d S^{+}\right)
$$

where

$$
S=\sum_{i=1}^{\infty} t_{i} K^{i}, \quad K^{n}=E\left(p, t_{1}, \ldots\right)
$$

$S^{+}$is the non-negative and $S^{-}$is the negative parts of (4.2). The polynomial $E\left(p, t_{1}, \ldots\right)$ is the solution of the dispersionless Lax hierarchy which was constructed in Sect. 2.

The residue of the differential is invariant with respect to change of variables

$$
K \rightarrow K+\varepsilon K^{m+1} \text {. }
$$

Hence,

$$
0=\delta_{m} F=-\frac{1}{2} \operatorname{res}\left(\sum_{i=1}^{\infty} i t_{i} K^{i+m} d S^{+}+S d \delta_{m} S^{+}\right) .
$$

For $m=-n, 0, n, 2 n, \ldots$ from the defining relations (2.17) it follows that the variation

$$
\delta_{m} S^{+}=n \frac{d S^{+}}{d E} \cdot E^{\frac{m+n}{n}}
$$

is an entire function of the variable $p$. Therefore

$$
\delta_{m} S^{+}=\sum_{i=1}^{\infty}\left(t_{i}\left(i\left[K^{i+m}\right]_{+}-\sum_{j=1}^{m} j \chi_{i j}\left[K^{m-j}\right]_{+}\right),\right.
$$


where $\chi_{i j}$ are coefficients of the expansion

$$
\left[K^{i}\right]_{+}=K^{i}+\sum_{j=1}^{\infty} \chi_{i j} K^{-j} .
$$

The equality (3.31) in our "new-old" times has the form

Hence

$$
\partial_{i} F=\operatorname{res}\left(S^{+} d K^{i}\right) \text {. }
$$

$$
\partial_{i} \partial_{j} F=\operatorname{res}\left(\left[K^{i}\right]_{+} d K^{j}\right)=j \chi_{i j}
$$

The substitution of (4.7) and (4.5) into (4.3) gives for $m=0, n, 2 n, \ldots$

$$
0=\sum_{i=1}^{\infty}\left[i t_{i} \partial_{i+m} F+\frac{1}{2} \sum_{j=1}^{m-1}\left(t_{i} \partial_{i} \partial_{j} F\right)\left(\partial_{m-j} F\right)\right] \text {. }
$$

From (4.7) and the definition of $F$ it follows

$$
\sum_{i=1}^{\infty} t_{i} \partial_{i} F=2 F
$$

Therefore

$$
\sum_{i=1}^{\infty} t_{i} \partial_{i} \partial_{j} F=\partial_{j}\left(\sum_{i=1}^{\infty} t_{i} \partial_{l} F\right)-\partial_{j} F=\partial_{j} F,
$$

and we obtain finally

$$
\sum_{i=1}^{\infty} i t_{i} \partial_{i+m} F+\frac{1}{2} \sum_{j=1}^{m-1} \partial_{j} F \partial_{m-j} F=0
$$

For $m=-n$,

$$
0=\delta F=\operatorname{res} \sum_{i=1}^{\infty} i t_{i}\left(K^{i+m} d S^{+}+S d\left[K^{i+m}\right]_{+}\right) .
$$

Using (3.31), we can represent the right-hand side of (4.13) in the form

$$
0=-2 \sum_{i=-m+1}^{\infty} i t_{i} \partial_{i+m} F+\operatorname{res}\left(\sum_{i=1}^{-m} i t_{i} K^{i+m} d S^{+}\right) \text {. }
$$

The second term in (4.14) equals

$$
\operatorname{res}\left(\sum_{i=1}^{-m} i t_{i} K^{i+m} d S\right)=-\sum_{i+j=-m} i j t_{i} t_{j},
$$

The differential operators

$$
L_{n}=\frac{1}{2} \sum_{i+j=-m} i j t_{i} t_{j}+\sum_{i-j=-m} i t_{i} \partial_{j}+\frac{1}{2} \sum_{i+j=m} \partial_{i} \partial_{j}
$$

are the generators of the Virasoro algebra with central charge $c=1$,

$$
\left[L_{n}, L_{m}\right]=(n-m) L_{n+m}+\frac{1}{12}\left(n^{3}-n\right) \delta_{n, 0} .
$$

From (4.14) and (4.15) it follows that the function

$$
\tau_{K}\left(t_{1}, \ldots\right)=\exp F
$$


satisfies the Virasoro constraints

$$
L_{m} \tau_{K}=0, \quad m=0,-n .
$$

The equalities (4.12) are the truncated versions of Virasoro constraints for $m>0$. This $\tau_{\kappa}$ should be an analogue of the usual $\tau$ in the theory of KP hierarchy.

In the next paper we consider the general algebraic nature of a general $\tau_{\kappa}$ function for the dispersionless integrable equations. For $\tau_{\kappa}$ which was constructed in this section (4.8) for $i=1$ is the relation

$$
\partial_{1} \partial_{m} \ln \tau_{k}=\operatorname{res}\left(K^{m} d p\right)
$$

which is the dispersionless analogue of the well-known relation for KP theory.

\section{The Khokhlov-Zabolotskaya Hierarchy}

The algebraic-geometrical construction of exact solutions of the two-dimensional KP-type integrable equations was proposed in [12]. In the generic case these solutions are quasi-periodic and can be expressed in terms of the Riemann thetafunction of auxiliary Riemann surfaces.

For example, for the KP-equation itself

$$
\frac{3}{4} u_{y y}=\left(u_{t}-\frac{3}{2} u u_{x}+\frac{1}{4} u_{x x x}\right)_{x}
$$

the exact solutions have the form

$$
u(x, y, t)=2 \partial_{x}^{2} \ln \theta(U x+V y+W t+Z / B),
$$

where $\theta\left(z_{1}, \ldots, z_{g} / B\right)$ is the Riemann theta-function which is constructed with the help of the matrix of $b$-periods of normalized holomorphic differentials on the Riemann surface $\Gamma_{g}$ of genus $g ; U, V, W$ are the vectors of $b$-periods of second kind differentials on $\Gamma_{g}$ which have a pole of the order $2,3,4$, respectively at the fixed point $P_{0}$ of $\Gamma_{g}$ and are holomorphic everywhere else.

The Whitham averaging method (or non-linear WKB-method) is the generalization for the evolution partial differential equations $(1+1$-systems) of the classical Bogolybov-Krylov method. It can be applied to any equation which has the set of exact solutions of the form

$$
u(x, t)=u_{0}\left(U x+W t / l_{1}, \ldots, l_{N}\right),
$$

where $u_{0}\left(z_{1}, \ldots, z_{g} / l_{1}, \ldots, l_{N}\right)$ is a periodic function of variables $z_{i}$ and depends on the parameters $l_{j}$. The vectors $U=U(l), W=W(l)$ are also functions of the same parameters.

The solutions of the form (5.3) can be used for the construction of asymptotic solutions for a perturbed equation or for the initial equation if one needs to solve it with non-trivial boundary conditions. The leading term of these asymptotic solutions has the form

$$
u=u_{0}\left(\varepsilon^{-1} S(X, T) / l(X, T)\right)+\varepsilon u_{1}+\ldots,
$$

where $X=\varepsilon x, T=\varepsilon t$ are the slow variables, and the vector-function $S$ is defined from the relations

$$
\partial_{x} S=U(X, T), \quad \partial_{t} S(X, T)=W(X, T) .
$$


It turns out that "self-consistency" of the expansion (5.3) requires that a dependence of the parameters $l(X, T)$ should be described by some partial differential equations. Any type of such equations are called by Whitham equations (see $[13,11]$ ).

In [11] the ideas of the Whitham method were generalized for the twodimensional integrable equations of the KP-type. The set of the parameters for the whole KP-hierarchy is: the algebraic curve $\Gamma$ with the fixed point $P_{0}$ and a local parameter $k^{-1}(P), k^{-1}\left(P_{0}\right)=0$, in the neighbourhood of $P_{0}$,

$$
\hat{M}=\left(\Gamma, P_{0}, k^{-1}\right) \text {. }
$$

The Whitham equations on these parameters was obtained in [11] in the following form:

For any set of data (5.5) let's define on the corresponding curve $\Gamma$ the meromorphic differentials $d \Omega_{j}, j=1,2,3, \ldots$, with the help of the conditions:

1. $d \Omega_{j}$ has the only pole at $P_{0}$ and its expansion in the neighbourhood of this is as follows:

$$
d \Omega_{j}=d k^{j}\left(1+O\left(k^{-j-1}\right)\right) .
$$

2. The differential $d \Omega_{j}$ has pure real periods on $\Gamma$

$$
\operatorname{Im} \int_{C} d \Omega_{j}=0, \quad C \in H_{1}(\Gamma)
$$

[the conditions $(5.6,5.7)$ define $d \Omega_{j}$ uniquely].

The equations

$$
d p\left(\partial_{i} \Omega_{j}-\partial_{j} \Omega_{i}\right)-d \Omega_{j}\left(\partial_{i} p-\partial_{x} \Omega_{i}\right)+d \Omega_{i}\left(\partial_{j} p-\partial_{x} \Omega_{j}\right)=0
$$

are equivalent to the full set of the equations on the parameters (5.5) and are necessary for the existence of asymptotic solutions of the KP-hierarchy with a uniformly bounded first correction term [11]. In (5.8) $d p=d \Omega_{1}, x=t_{1} ; \Omega_{j}$-are the multi-valued functions.

In [11] the construction of the exact solutions of (5.8) was proposed. A particular case of this construction has been used in Sect. 2. We would like to mention that in the case of perturbed KP-hierarchy the right-hand side of (5.8) contains an additional term. This more general equation was used in [14] for the Douglas equations.

The subsets $M_{g}$ of the data (5.5) corresponding to the curves $\Gamma_{g}$ of the fixed genus $g$ are invariant with respect to Eqs. (5.8). In the case $g=0$ the local parameter $k^{-1}$ is the only part of the data (5.5). Hence, Eqs. (5.8) give the hierarchy of nonlinear equations on the coefficients of the series:

$$
K(p)=p+\xi_{1} p^{-1}+\xi_{2} p^{-2}+\ldots
$$

In the case $g=0$ there are no conditions (5.7). The integrals of the differentials $d \Omega_{j}$ have to be polynomials. Therefore, from (5.6) we have

$$
\Omega_{j}(p)=\left[K^{j}(p)\right]_{+} .
$$

The Important Remark. The equations (5.8) are invariant with respect to a change of parameter. It means that $\Omega_{j}$ can be considered as the functions of any local parameter $z, p=p(z)$. The derivatives with respect to $t_{i}$ are taken for the fixed $z$. Any change of local parameter $z$ preserves the form of Eq. (5.8). 
The first polynomials have the form

$$
\Omega_{2}=p^{2}+u, \quad \Omega_{3}=p^{3}+\frac{3}{2} p+w,
$$

where

$$
u=2 \xi_{1}, \quad w=3 \xi_{2} .
$$

The substitution of (5.11) into (5.8) gives

$$
w_{x}=\frac{3}{4} u_{y}, \quad w_{y}=u_{t}-\frac{3}{2} u u_{x},
$$

which implies the Khokhlov-Zabolotskaya equation

$$
\frac{3}{4} u_{y y}=\left(u_{t}-\frac{3}{2} u u_{x}\right)_{x} .
$$

The Eqs. (5.8) are the dispersionless analogue of the zero-curvature representation of the KP-hierarchy. Some of their solutions can be described with the help of evolution equations which are the analogue of the Sato hierarchy.

The analogue of the Sato representation for the KP-hierarchy is evolution equations for the local parameter $K(p)$ which have the form

$$
\partial_{i} K(p)=\frac{d \Omega_{i}}{d p} \partial_{x} K-\frac{d K}{d p} \partial_{x} \Omega_{i}
$$

It should be mentioned that (5.14) has the Hamiltonian form. The right-hand side of it formally is equal to the canonical bracket for the functions depending on two variables $p, x$.

The right-hand side of (5.14) equals

$$
\frac{d K}{d p} \partial_{x}\left[K^{i}\right]_{-}-\frac{d\left[K^{i}\right]}{d p} \partial_{x} K,
$$

which proves that Eqs. (5.14) are well-defined.

Acknowledgements. The author would like to acknowledge discussions with C. Vafa. I thank the Scuola Normale in Pisa and the Institute for Scientific Interchange in Turin where most of this work was done.

\section{References}

1. Eguchi, T., Yang, S.-K.: $N=2$ Superconformal models as topological field theories. Tokyo preprint UT-564

2. Witten, E.: Commun. Math. Phys. 118, 411 (1988)

3. Vafa, C., Warner, N.P.: Phys. Lett. 218 B, 51 (1989);

Lerche, W., Vafa, C., Warner, N.P.: Nucl. Phys. B 324, 427 (1989);

Martinec, E.: Phys. Lett. 217 B, 431 (1989); Criticality, catastrophe and compactifications. V.G. Knizhnik memorial volume, 1989

4. Li, K.: Topological gravity with minimal matter. Caltech preprint CALT-68-1662

$\mathrm{Li}, \mathrm{K}$.: Recursion relations in topological gravity with minimal matter. Caltech preprint CALT-68-1670

5. Dijkgraaf, R., Witten, E.: Nucl. Phys. B 342, 486 (1990)

6. Witten, E.: Commun. Math. Phys. 117, 353 (1988)

7. Gross, D., Migdal, A.: Nucl. Phys. B 340, 333 (1990)

8. Banks, T., Douglas, M., Seiberg, N., Shenker, S.: Phys. Lett. 238 B, 279 (1990) 
9. Douglas, M.: Phys. Lett. 238 B, 176 (1990)

10. Dijkgraaf, R., Verlinde, H., Verlinde, E.: Topological strings in $d=1$. Princeton preprint PUPT-1204, IASSNS-HEP-90/71

11. Krichever, I.M.: Funct. Anal. Appl. 22, No. 3, 37 (1988)

Krichever, I.M.: Uspekhi Mat. Nauk 44, No. 2, 121 (1989)

12. Krichever, I.M.: Sov. Dokl. 227, No. 2, 291 (1976)

Krichever, I.M.: Funct. Anal. Appl. 11, No. 3, 15 (1977)

13. Dobrokhotov, S.Yu., Maslov, V.P.: Sov. Scientific Reviews. Math. Phys. Rev. 3, 221 (1982): OPA Amsterdam

Flashka, H., Forest, M., McLaughlin, D.: Commun. Pure and Appl. Math. 33, No. 6, 739 (1980)

14. Krichever, I.M.: The Hiezenber relations for ordinary differential operators. Preprint ETH Zürich, 1990

15. Zakharov, V.E.: Funk. Anal. Appl. 14, 89 (1980)

16. Kodama, Y., Gibbons, J.: Integrability of the dispersionless KP hierarchy. Proceedings of Workshop on Nonlinear and Turbulent Processes in Physics. Kiev, 1989

Communicated by Ya. G. Sinai 
